\title{
Temperaturas de germinación de cuatro gramíneas nativas del pastizal halofítico de la Pampa Deprimida, Argentina
}

\author{
Víctor R. A. Bolaños ${ }^{12,2}$; María C. Vecchio ${ }^{1}$ \& Rodolfo A. Golluscio ${ }^{3 / 4}$ \\ ${ }^{1}$ Universidad Nacional de La Plata. Facultad de Ciencias Agrarias y Forestales. Cátedra de Forrajicultura y Praticultura. \\ Buenos Aires, La Plata, Argentina. ${ }^{2}$ CONICET. La Plata, Buenos Aires, Argentina. ${ }^{3}$ Universidad de Buenos Aires. Facultad \\ de Agronomía. Cátedra de Forrajicultura. Buenos Aires, Argentina. ${ }^{4}$ Universidad de Buenos Aires - CONICET. Instituto \\ de Investigaciones Fisiológicas y Ecológicas Vinculadas a la Agricultura (IFEVA). Facultad de Agronomía. Buenos Aires, \\ Argentina.
}

\begin{abstract}
Resumen. Se evaluó el comportamiento germinativo de cuatro gramíneas nativas con valor forrajero propias de la estepa de halófitas del pastizal de la Pampa Deprimida, bajo tres temperaturas de germinación constantes y un régimen de amplitud térmica elevada. Tres de las especies tienen crecimiento estival. Stapfochloa berroi Arech. y Sporobolus indicus Br. son perennes, y Leptochloa fusca ssp. uninervia N. Snow es anual. Por su parte, Polypogon elongatus Kunth es invernal y perenne. Nueve meses después de recolectadas, se sembraron sobre papel de filtro cincuenta simientes seleccionadas por unidad experimental. En incubadora se ensayaron tres temperaturas constantes: $25^{\circ} \mathrm{C}, 28^{\circ} \mathrm{C}, 37^{\circ} \mathrm{C}$, y un régimen de temperatura con alternancia: $12 \mathrm{hs}$ a $20^{\circ} \mathrm{C}$ y 12 hs a $35^{\circ} \mathrm{C}(\mathrm{TA})$. Luz y humedad permanecieron constantes. Se usó un DCA con arreglo factorial (especies $\mathrm{x}$ temperatura) con cinco repeticiones. Se contabilizó el número de simientes germinadas a los 5, 10, 18 y 25 días. Se calculó el poder germinativo (PG) en proporción a las semillas viables y, además, se evaluó la energía germinativa mediante el gráfico de la curva de evolución del PG y el cálculo del índice de velocidad de germinación. Los máximos PG de las tres especies estivales evidencian que, al momento, del ensayo gran parte de la dormición había disminuido, y no sucedió así en la invernal. Tanto S. berroi como S. indicus sólo registraron máximo PG y energía germinativa bajo TA. A diferencia de las perennes, L. fusca ssp. uninervia mostró máximos valores tanto en TA como a $37^{\circ} \mathrm{C}$. A diferencia de las estivales, P. elongatus tuvo máximo PG a $25^{\circ} \mathrm{C}$ y no germinó en TA. Los requerimientos de cada especie para germinar se podrían interpretar como ejemplos de adaptación ecológica al ambiente; este conocimiento podría ser usado para ensayar modos de utilización del pastizal natural que las promocione.
\end{abstract}

[Palabras clave: estepa de halófitas, especies forrajeras, requerimientos térmicos de germinación]

\begin{abstract}
AвSTRACT. Germination temperatures of four native grasses of halophytic grassland of the Flooding Pampas, Argentina. The germination of four grasses with fodder value and native from the halophytic steep of the grassland of the flooding Pampa, was evaluated under four temperatures regimens. Three have summer growth. Among them, Stapfochloa berroi Arech. and Sporobolus indicus Br. are perennials, while Leptochloa fusca ssp. uninervia N. Snow is annual and Polypogon elongatus Kunth is wintry and perennial. Nine months after harvested, fifty selected seeds by experiment unit were sown on filter paper. On incubator three constant temperatures were evaluated: $25^{\circ} \mathrm{C}, 28^{\circ} \mathrm{C}, 37^{\circ} \mathrm{C}$, and an alternant temperature regime: 12 hours at $20^{\circ} \mathrm{C}$ and 12 hours at $35^{\circ} \mathrm{C}$ (TA). Light and humidity remained constant. A CRD with factorial arrangement was used (species $\mathrm{x}$ temperature), with five repetitions. The number of germinated seeds was counted at days 5, 10, 18 and 25 . The germination power in proportion to the viable seeds was calculated (PG) and, in addition, the germinative energy was evaluated using the curve of PG evolution and the calculus of the germination speed index. The maximum PG of the three summer species evidenced that, at the moment of the experiment, a big part of the dormancy had diminished, not so the wintry one. Both S. berroi and S. indicus only registered a maximum PG and more germinative energy under TA, while, in difference to the perennials, L. fusca ssp. uninervia showed maximum values both at $\mathrm{TA}$ and $37^{\circ} \mathrm{C}$. In difference to the summer ones, P. elongatus had a maximum PG at $25^{\circ} \mathrm{C}$ and did not germinate in TA. Each species' requirements to germinate might be interpreted as examples of ecological adaptation to the environment; this knowledge can be used to test different ways of using natural grasslands that promote them.
\end{abstract}

[Keywords: the halophytic steep, fodder species, thermic requirement of germination.

Editora asociada: Adriana Salvo
Recibido: 23 de Julio de 2018

Aceptado: 5 de Febrero de 2019 


\section{INTRODUCCIÓN}

La Pampa Deprimida (Cabrera 1994) conserva una de las áreas más grandes de pastizales naturales del mundo. En esta región, el pastizal desempeña un rol económico importante como principal sustento forrajero en la producción de cría bovina (Rearte 1997; Cauhépé and Hidalgo 2005), además de un papel ecológico clave como proveedor de servicios intangibles (Soriano 1991). El pastizal de la Pampa Deprimida está constituido por un mosaico de estepas y praderas graminosas con gran heterogeneidad florística (Vervoorst 1967; León et al. 1979; Batista et al. 1988; Burkart et al. 1990; Burkart et al. 1998). Tal diversidad de comunidades vegetales fue clasificada en cinco grandes unidades de vegetación asociadas con la posición topográfica, el régimen hídrico y la salinidad o la alcalinidad de los suelos (Perelman et al. 1982; Batista et al. 1988; Perelman et al. 2001). Una de esas unidades de vegetación es la estepa de halófitas. Representa $\sim 23 \%$ de la superficie del pastizal (Burkart et al. 2005), su cobertura vegetal es por lo general rala (León et al. 1979; Perelman et al. 2001; Burkart et al. 2005) y se desarrolla sobre los suelos halomórficos ubicados en las posiciones bajas del relieve y propensas a anegarse. Se desarrolla sobre suelos sódicos, con bajo contenido de materia orgánica, pobre infiltración y problemas de anegamiento intercalados con déficits hídricos durante el verano. En consecuencia, la estepa de halófitas es uno de los ambientes del pastizal con mayores restricciones para el establecimiento de especies exóticas o nativas propias de ambientes más fértiles (Chaneton et al. 2002; Puhl et al. 2014).

El efecto del pastoreo de ganado doméstico sobre la vegetación y el suelo en la estepa de halófitas difiere (y en cierto caso se contrapone) del que tiene lugar en otros ambientes del mismo pastizal (Chaneton et al. 2002; Puhl et al. 2014). En términos de cobertura vegetal se reportó un aumento significativo en el porcentaje de suelo desnudo en estepas de halófitas con larga historia de pastoreo continuo, explicado por una fuerte disminución en la cobertura vegetal y en la broza (Vecchio et al. 2019). Asimismo, pero en términos edáficos, se encontró una mayor alcalinidad y menores valores de materia orgánica, nitrógeno total, tasa de infiltración y estabilidad estructural en estepas sometidas a pastoreo continuo con porcentajes de suelo desnudo mayores al 50\% (Vecchio et al. en prensa b). La presencia de cobertura es de vital importancia en los suelos halomórficos, dado que modera los regímenes hídrico y térmico del suelo (Phillips 1984), y el riesgo de salinización o alcalinización por acenso y acumulación de sales o sodio (Lavado and Taboada 1987). Se ha documentado que la mayor producción de biomasa vegetal en estepas de halófitas conlleva a una mayor cobertura y, finalmente, a mejoras en las propiedades químicas del suelo (Otondo 2011; Vecchio et al. 2018), lo que sugiere que el aumento de la cobertura vegetal es un factor clave para el proceso de recuperación en estepas de halófitas degradadas por el sobrepastoreo de ganado doméstico. La reincorporación de las especies nativas de alto valor forrajero es la mejor alternativa para aumentar la cobertura y favorecer procesos de recuperación en dicho ambiente, ya que las condiciones ambientales resultan restrictivas para la mayoría de las especies cultivadas, mientras que las especies nativas están adaptadas a esas condiciones (Snyman 2003; Cole et al. 2005; Mollard 2007; Quiroga et al. 2009). Sin embargo, la reincorporación de las especies nativas requiere conocer sus aspectos biológicos y ecológicos; esta información es escasa o aún inexistente para la mayoría de las forrajeras nativas de la estepa de halófitas del pastizal natural de la Pampa Deprimida.

La germinación es un aspecto central de la biología de las especies y resulta de vital importancia para que las poblaciones se mantengan y recuperen (Rees 1997). Para que una semilla germine requiere que se cumpla un conjunto de condiciones externas e internas (Rees 1997; Probert 2000). En cuanto a las condiciones externas o del ambiente, se sabe que cuando la humedad es adecuada, la temperatura controla mayormente la germinación (Bierhuizen and Wagenvoort 1974). En relación con las condiciones internas de la semilla, la presencia de dormición puede impedir la germinación aun bajo condiciones externas óptimas (Baskin and Baskin 1998). Es frecuente que las semillas de las gramíneas forrajeras no domesticadas posean algún tipo de dormición y que dicho fenómeno esté regulado por un determinado requerimiento pregerminativo que difiere entre especies (Shaidaee et al. 1969). Existen diversos tratamientos que se aplican a las semillas antes de ser sembradas, como la estratificación en frio o el pelado manual de las cubiertas que recubren la cariópside, a fin de romper su dormición (Mollard 2007). Asimismo, 
bajo condiciones naturales, la dormición de las semillas dispersadas se va morigerando gradualmente, por lo que, para cada especie, el fenómeno tiene una perdurabilidad más o menos definida (Bierhuizen and Wagenvoort 1974). En tal sentido, un período postcosecha de ocho meses resultó suficiente para restar gran parte de la dormición de las espiguillas de Stapfochloa berroi Arechav. (Bolaños et al. 2015), Sporobolus indicus (L.) R. Br. (Ferrari and Rossi 1997) y Polypogon imberbis (Phil) Johow (Ferrari 1999), y uno de doce meses para Leptochloa fusca (L.) Kunth (Morgan and Myers 1989).

Parte de la información que se necesita para caracterizar las condiciones óptimas de germinación de las principales especies forrajeras nativas no domesticadas, es conocer los requerimientos térmicos de germinación; esto permite inferir el momento del año en el que se maximiza la germinación a campo y planificar un manejo estratégico del pastoreo que favorezca la regeneración de las poblaciones vegetales. Cada especie tiene una temperatura óptima de germinación a la que tiene lugar el porcentaje más alto de plántulas normales y se alcanza en el menor tiempo (Mayer and Poljakoff-Mayber 1989). Para el caso de las gramíneas forrajeras tropicales, la temperatura óptima de germinación se suele encontrar a $\sim 35{ }^{\circ} \mathrm{C}$ (Yoon et al. 1985). Sin embargo, la información de los requerimientos térmicos para germinar es escasa o imprecisa para la mayoría de las especies no domesticadas del pastizal de la Pampa Deprimida. En su estudio, Ferrari y Rossi (1997) evaluaron la germinación de cinco especies nativas de dicho pastizal, entre ellas Sporobolus indicus y Polypogon imberbis, bajo seis condiciones de temperatura alterna $(15 /$ $10,20 / 10,20 / 15,20 / 25,20 / 30$ y $20 / 35^{\circ} \mathrm{C}$, con períodos de $8 / 16$ horas) y dos de temperatura constante (15 y $\left.20^{\circ} \mathrm{C}\right)$, y encontraron que la germinación de ambas especies fue favorecida por la alternancia; en el caso de $S$. indicus, por la alternancia $20 / 35{ }^{\circ} \mathrm{C}$; en el caso de $P$. imberbis, por la de $20 / 25^{\circ} \mathrm{C}$. No obstante, al haber utilizado dos temperaturas constantes relativamente bajas para gramíneas de ciclo estival no queda claro si la mejor germinación de $S$. indicus en condiciones de alternancia se debió a un requerimiento de la especie o a que las temperaturas constantes ensayadas resultaron bajas o subóptimas.

Las especies ensayadas en este trabajo fueron Stapfochloa berroi (Arechav.) P. M. Peterson, Sporobolus indicus (L.) R. Br., Leptochloa fusca ssp. uninervia (J. Presl.) N. Snow y Polypogon elongatus Kunth. Todas ellas son especies nativas. En el pastizal de la Pampa Deprimida se las reconoce como propias de la gran unidad de vegetación estepa de halófitas (León 1979; León et al. 1984; Perelman et al. 2001). La distribución de estas especies a nivel provincial dentro del territorio argentino es bastante extensa, e incluso en países limítrofes (Figura 1). S. indicus y P. elongatus se encuentran distribuidas desde las provincias argentinas más nórdicas (como Jujuy y Misiones) hasta la provincia de La Pampa (en el caso de la primera) y de Río Negro (en el caso de la segunda). Las dos se encuentran en Brasil, Paraguay, Uruguay y en Chile. $P$. elongatus, L. fusca ssp. uninervia fue descripta en las provincias de Buenos Aires, Entre Ríos, La Rioja, Misiones, Río Negro, San Juan y en el Alto Paraguay (Zuloaga et al. 2008). En el caso de $S$. berroi no se encontró una referencia de su distribución, pero fue descripta en la provincia de Buenos Aires (desde el Partido de Magdalena hacia el norte [Rolando J. C. León, comunicación personal] y en la provincia de Entre Ríos. En Uruguay fue descrita en los partidos de Montevideo, Paysandú, Río Negro, San José y Soriano. Las cuatro especies tienen gran valor forrajero tanto por sus propiedades nutricionales - según el índice de calidad propuesto por Cauhépé et al. (1985)— como por su capacidad para crecer y desarrollarse en ambientes con limitaciones edáficas como el estrés hídrico y la alcalinidad por sodio. Tanto S. berroi como S. indicus y L. fusca ssp. uninervia poseen síndrome fotosintético $\mathrm{C}_{4} \mathrm{y}$ crecimiento estival, pero $L$. fusca ssp. uninervia es anual, mientras que las dos primeras son perennes. Por su parte, P. elongatus es perenne, con síndrome fotosintético $\mathrm{C}_{3} \mathrm{y}$ crecimiento invernal. Se reportó que el sobrepastoreo del ganado doméstico afecta de forma negativa a estas especies, ya que disminuye el número de plantas hasta, incluso, hacerlas desaparecer de la comunidad vegetal (Vecchio et al. a, en prensa). Si bien existen evidencias del efecto negativo que el pastoreo causa sobre las especies nativas con valor forrajero $y$, en consecuencia, sobre la cantidad y calidad de forraje que ofrece la comunidad natural, en la bibliografía no se hallaron trabajos que evalúen formas de manejo del pastoreo que favorezcan la presencia de tales especies. En suma, la información disponible respecto a aspectos básicos de la ecología y la fisiología de la mayoría de las especies nativas con valor forrajero es insuficiente $\mathrm{y}$, en algunos casos, inexistente. El objetivo de este trabajo 
es evaluar el comportamiento germinativo de cuatro gramíneas nativas con valor forrajero propias de la estepa de halófitas del pastizal de la Pampa Deprimida, ensayando tres temperaturas de germinación constantes y un régimen elevado de amplitud térmica.

\section{Materiales y MétOdOS}

Se analizó la dinámica de la germinación de cuatro gramíneas nativas propias de la comunidad estepa de halófitas del pastizal de la Pampa Deprimida, en respuesta a tres temperaturas constantes y un régimen térmico con alta amplitud. Las semillas fueron recolectadas entre diciembre de 2015 y marzo de 2016 en un pastizal ubicado en la localidad de Vieytes, Buenos Aires, Argentina (Figura 1). En cada cosecha se seleccionaron las espigas que presentaban coloración pajiza, dehiscencia de espiguillas y un tono castaño en la porción de la caña próxima a la espiga. Estos atributos visuales se usaron previamente en Bromus auleticus Trin. para identificar a campo espigas con cariópsides fisiológicamente maduros (Ruiz et al. 2003). Las espigas cosechadas fueron acondicionadas, se separaron las espiguillas y se descartaron los restos de la inflorescencia.
Las espiguillas limpias (cariópsides cubierto por las glumelas) se colocaron sobre bandejas durante siete días para que se orearan y luego dentro de bolsas de papel madera por un período de 8 a 12 meses, aproximadamente a 20-25 ${ }^{\circ} \mathrm{C}$ de temperatura y $40-60 \%$ de humedad relativa ambiente.

En el caso de Sporobolus indicus se usaron cariópsides y en los de S. berroi, L. fusca ssp. uninervia y $P$. elongatus se usaron espiguillas, dado que son la forma en que naturalmente dispersan las simientes cada especie. Se eligieron las cariópsides de color castaño, brillantes y turgentes, y las espiguillas que presionadas con la yema del dedo contra la mesada demostraban no ser vanas. Tanto las cariópsides como las espiguillas seleccionadas, nombradas de aquí en más como simientes seleccionadas, se sembraron sobre papel de filtro acondicionado dentro de bandejas plásticas circulares con tapa (12 $\mathrm{cm}$ de diámetro y $6 \mathrm{~cm}$ de altura). Por debajo del papel se colocó una capa de algodón (2-3 cm altura) a fin de conservar mejor la humedad. La siembra se hizo de forma manual, acomodando dentro cada bandeja cincuenta simientes seleccionadas, en cinco filas de diez.

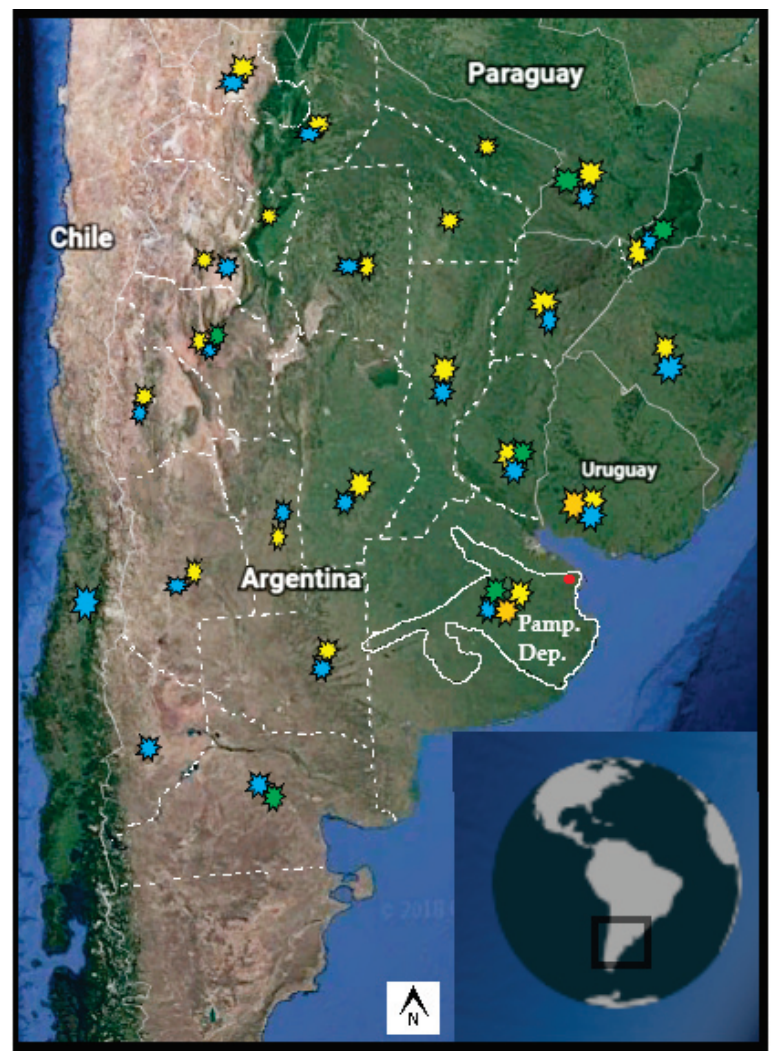

Figura 1. Distribución geográfica de las cuatro especies en estudio, a nivel provincial dentro del territorio argentino y en países limítrofes. Las estrellas de varios puntos indican la provincia argentina o país limítrofe donde fue descripta la especie. El color naranja indica Stapfochloa berroi (Arechav.) P. M. Peterson; amarillo indica a Sporobolus indicus (L.) R. Br.; verde, a Leptochloa fusca ssp. uninervia (J. Presl.) N. Snow; azul indica a Polypogon elongatus Kunth. El punto rojo dentro de la fito-región Pampa Deprimida (Pamp. Dep.) indica la localidad de donde fueron recolectadas las semillas. Localidad de Vieytes, Buenos Aires, Argentina. Fuentes: Zuloaga et al. 2008, www.darwin.edu.ar e información personal.

Figure 1. Geographic distribution of the four species under study, at provincial level within the Argentinian territory and in bordering countries. The stars of several points indicate the Argentinian province or bordering country where the species was described. Orange color indicates Stapfochloa berroi (Arechav.) P. M. Peterson; yellow, Sporobolus indicus (L.) R. Br.; green, Leptochloa fusca ssp. uninervia (J. Presl.) N. Snow; blue, Polypogon elongatus Kunth. The red point within the Pampa Deprimida phytoregion (Pamp. Dep.) indicates the location from which the seeds were collected. Locality of Vieytes, Buenos Aires, Argentina. Sources: Zuloaga et al. 2008, www.darwin.edu.ar and personal information. 


\section{Diseño experimental}

Se utilizó un diseño completamente aleatorizado con arreglo factorial (especies $x$ temperatura), con cuatro niveles para cada factor y cinco repeticiones (la unidad experimental fue cada bandeja). Se emplearon tres temperaturas constantes $\left(\mathrm{TC} \pm 2{ }^{\circ} \mathrm{C}\right): \mathrm{TC}_{1}$ $25^{\circ} \mathrm{C}, \mathrm{TC}_{2} 28^{\circ} \mathrm{C}, \mathrm{TC}_{3} 37^{\circ} \mathrm{C}$ y un régimen de temperatura con alta amplitud térmica: TA $20 \mathrm{y}$ $35^{\circ} \mathrm{C}$ en períodos de 12 horas y 12 horas. Dichos tratamientos fueron seleccionados en función de la mejor temperatura de germinación reportada en la bibliografía para gramíneas estivales, $35{ }^{\circ} \mathrm{C}$ (Yoon et al. 1985), de datos reportados para $S$. indicus y Polypogon imberbis (Ferrari and Rossi 1997) y a información propia de datos no publicados obtenidos a partir de ensayos previos. Respecto a la luz y la humedad, permanecieron constantes durante todo el ensayo. Se usaron simultáneamente cuatro cámaras de germinación, todas con la misma fuente de luz (3x Philips Lifemax Tubelight, $725 \mathrm{~lm}$ cool daylight). La humedad se reguló mediante riegos periódicos con agua bidestilada. Además, cada cámara contaba, a modo de control, con un datalogger que registraba la temperatura cada 5 horas y que, al final del ensayo constató la regularidad de la temperatura programada en cada cámara.

\section{Registro de datos}

A $\operatorname{los} 5,10,18$ y 25 días postsiembra se contó el número de simientes germinadas, para lo que se consideró como tales aquellas con la radícula visible y el coleóptilo emergido, con la primera hoja sana. Las simientes con presencia de ennegrecimiento o que no llegaban a expandir su primera hoja se eliminaban de la bandeja y se contabilizaban como no viables. A las simientes que no germinaron al día 25 postsiembra se les determinó viabilidad mediante la prueba de tetrazolium (ISTA 1996). Esto permitió expresar los resultados de las variables de germinación en relación al total de semillas viables puestas a geminar en cada bandeja.

\section{Variables de germinación y análisis de datos}

Se calculó el poder germinativo (PG) como la sumatoria de simientes germinadas hasta el día 25 postsiembra en proporción al número de simientes viables colocadas en cada bandeja. Para evaluar la energía germinativa se graficó la curva de evolución del PG durante el período que comprendió el ensayo y se calculó el índice de velocidad de germinación
(IVG) propuesto por Maguire (1962). El IVG expresa la cantidad de semillas germinadas en relación con el número de días transcurridos desde la siembra. Su unidad se expresa en número de semillas germinadas por día y se calcula según la Ecuación 1

$$
\text { IVG }=\sum_{i=1}^{n} \frac{G_{i}}{t_{i}}
$$

donde $t_{i}$ es el número de días trascurridos desde el ínicio del ensayo hasta el momento del recuento iésimo y $G_{i}$ es el número de simientes germinadas en el recuento iésimo.

Para el análisis de los datos se utilizó el paquete estadístico InfoStat versión 1.1 (Di Rienzo et al. 2016). Previo al análisis de los datos se evaluó el cumplimiento de los supuestos de la distribución normal del error a partir de la prueba de Shapiro-Wilks y de homocedasticidad de la varianza con la prueba de Levene. En consecuencia, los análisis se realizaron sobre la proporción de las simientes germinadas con respecto a las viables (PG) y sobre la raíz cuadrada del IVG. La prueba de igualdad de medias se hizo mediante análisis de varianza (ANOVA). Para la comparación múltiple de las medias se utilizó la prueba de Tukey $(\alpha=0.05)$.

\section{Resultados}

La viabilidad de las simientes, expresada en proporción al número de simientes seleccionadas puestas a germinar, fue 100\% para Stapfochloa berroi; $96 \%$ para Sporobolus indicus; $100 \%$ para Leptochloa fusca ssp. uninervia y $84 \%$ para Polipogon elongatus. Por otra parte, como el análisis de la varianza mostró interacción temperatura $x$ especie significativa para todas las variables analizadas (PG: $\mathrm{F}=30.29, P=0.0001$; IVG: $\mathrm{F}=19.86, P<0.0001)$, la variación del comportamiento germinativo bajo las distintas temperaturas se evaluó de forma individual para cada especie.

\section{Poder germinativo (PG)}

El régimen de temperatura afectó significativamente $(P<0.05)$ el $P G$ en todas las especies. Stapfochloa berroi registró el máximo PG $(76 \pm 14 \%)$ bajo el régimen de temperatura con alta amplitud térmica (TA) y fue significativamente superior $(P<0.05)$ respecto a todas las temperaturas constantes (TC) ensayadas, entre las cuales la más alta $\left(\mathrm{TC}_{3}\right)$ presentó mayor PG $(44.4 \pm 6.5 \%)$ que las otras dos $\left(\mathrm{TC}_{2}: 6.4 \pm 4.3 \%\right.$ y $\mathrm{TC}_{1}: 6.3 \pm 3.3 \%$ ) (Figura 2). Por otro lado, Sporobolus indicus 


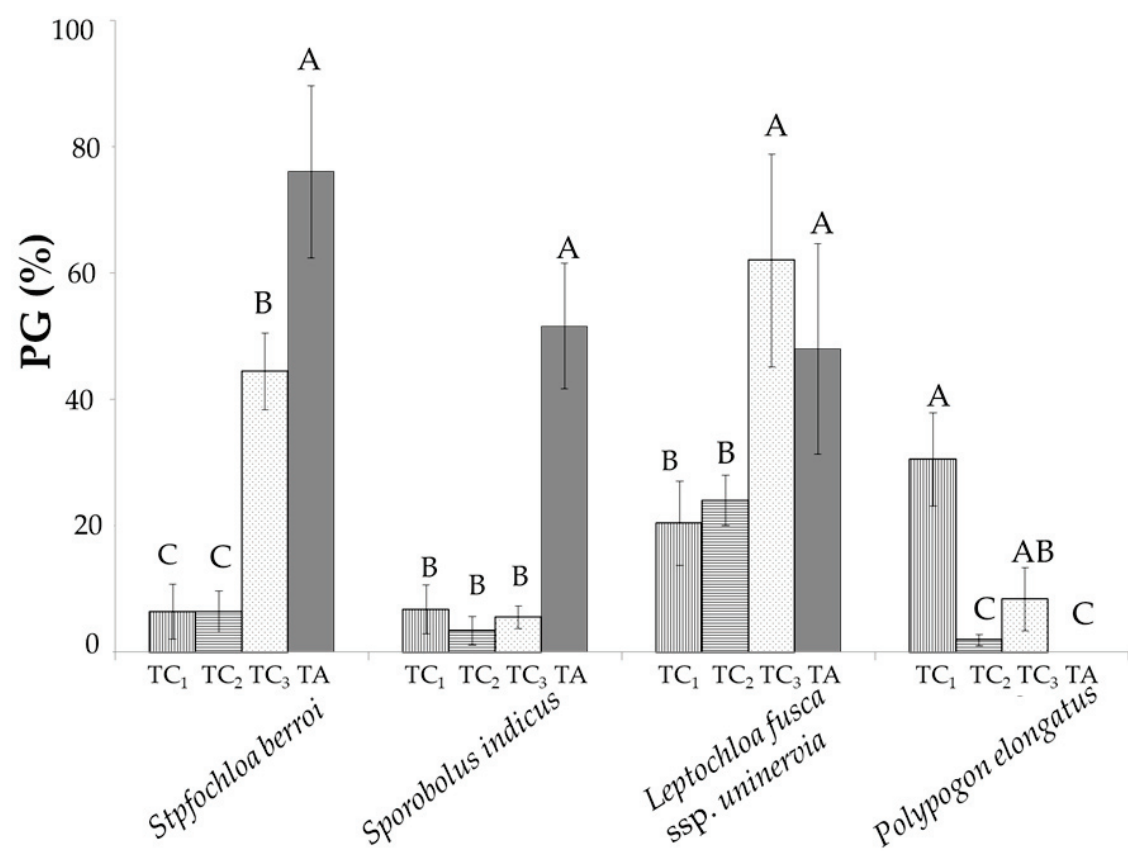

Figura 2. Porcentaje de germinación (PG) acumulada al día 25 postsiembra, expresada en proporción al número de semillas viables de cada especie puestas a germinar, bajo tres temperaturas constantes $\left(\mathrm{TC}_{1}: 25^{\circ} \mathrm{C}, \mathrm{TC}_{2}: 28^{\circ} \mathrm{C}, \mathrm{TC}_{3}: 37\right.$ ${ }^{\circ} \mathrm{C}$ ) y bajo un régimen de temperatura alterna (TA $20 / 35^{\circ} \mathrm{C}$ en períodos de 12 horas/12 horas). Las líneas verticales muestran el Desvío Estándar. Letras distintas indican diferencias significativas entre temperaturas dentro de cada especie (Tukey, $P<0.05$ ).

Figure 2. Germination percentage (PG) accumulated at day 25 post-sown expressed in proportion to the number of viable seeds of each species germinating under three constant temperatures $\left(\mathrm{TC}_{1}: 25^{\circ} \mathrm{C}_{,} \mathrm{TC}_{2}: 28{ }^{\circ} \mathrm{C}, \mathrm{TC}_{3}: 37^{\circ} \mathrm{C}\right)$ and under an alternant temperature regimen (TA $20 / 35^{\circ} \mathrm{C}$ in periods of 12 hours $/ 12$ hours). The vertical lines show the standard deviation. Different letters indicate significative differences between temperatures within each specie (Tukey, $P<0.05)$.

también registró mayor PG con el régimen TA $(51.6 \pm 11 \%)$, pero sin diferencias entre las TC (Figura 2). Por su parte, la especie anual Leptochloa fusca ssp. uninervia mostró valores de PG máximos tanto con el régimen de TA $(48 \pm 16.7 \%)$ como en la más cálida dentro de las TC ensayadas $\left(\mathrm{TC}_{3}: 62 \pm 18.6 \%\right.$ ), sin diferencia entre $\mathrm{TC}_{1}$ y $\mathrm{TC}_{2}$, en los que registró el menor PG (Figura 2). Contrariamente a lo hallado para las tres especies estivales, en el caso de Polipogon elongatus el mayor PG se registró en la TC más baja de las ensayadas (TC $130.5 \pm 7.4 \%$ ), bajo $\mathrm{TC}_{2}$ y $\mathrm{TC}_{3}$ los valores de PG fueron significativamente menores $\left(\mathrm{TC}_{2}: 1.9 \pm 0.9 \%\right.$ y $\left.\mathrm{TC}_{3}: 10.9 \pm 6 \%\right)$ sin diferencia entre ambos, y no registró germinación con TA (Figura 2).

\section{Energía germinativa}

La dinámica de la germinación fue distinta, tanto entre especies como entre temperaturas (Figura 3). Mientras la especie anual L. fusca ssp. uninervia al día 5 postsiembra registró, bajo todas las condiciones de temperatura, un único pulso germinativo, con valores más el- evados en $\mathrm{TC}_{3} \mathrm{y} \mathrm{TA}$, las tres especies perennes alcanzaron sus valores máximos de PG más tarde. En el caso de S. berroi, los incrementos también ocurrieron en la condición de TA y $\mathrm{TC}_{3}$, pero el máximo $\mathrm{PG}$ se dio recién al día 18 postsiembra; mientras tanto, $S$. indicus y $P$. elongatus lo alcanzaron al día 10 postsiembra, y sólo a TA en $S$. indicus y $\mathrm{TC}_{1}$ en $P$. elongatus (Figura 3).

En concordancia con los patrones descriptos, S. berroi y L. fusca ssp. uninervia tuvieron un mayor IVG $(P<0.05)$ a TA y TC ${ }_{3}$, sin diferencias entre sí ni entre $\mathrm{TC}_{1}$ y $\mathrm{TC}_{2}$ (Tabla1). En tanto, S. indicus mostró mayor IVG sólo a TA y $P$. elongatus a $\mathrm{TC}_{1}$ (Tabla 1$)$.

\section{DISCUSIÓN}

El poder germinativo elevado registrado en las especies estivales evidencia que ocho meses después de cosechadas habían perdido gran parte de la dormición, lo cual coincide con lo reportado por Morgan y Mayer (1989), Ferrari y Rossi (1997), Ferrari (2008) y Bolaños et al. (2015), quienes trabajaron con las mismas 


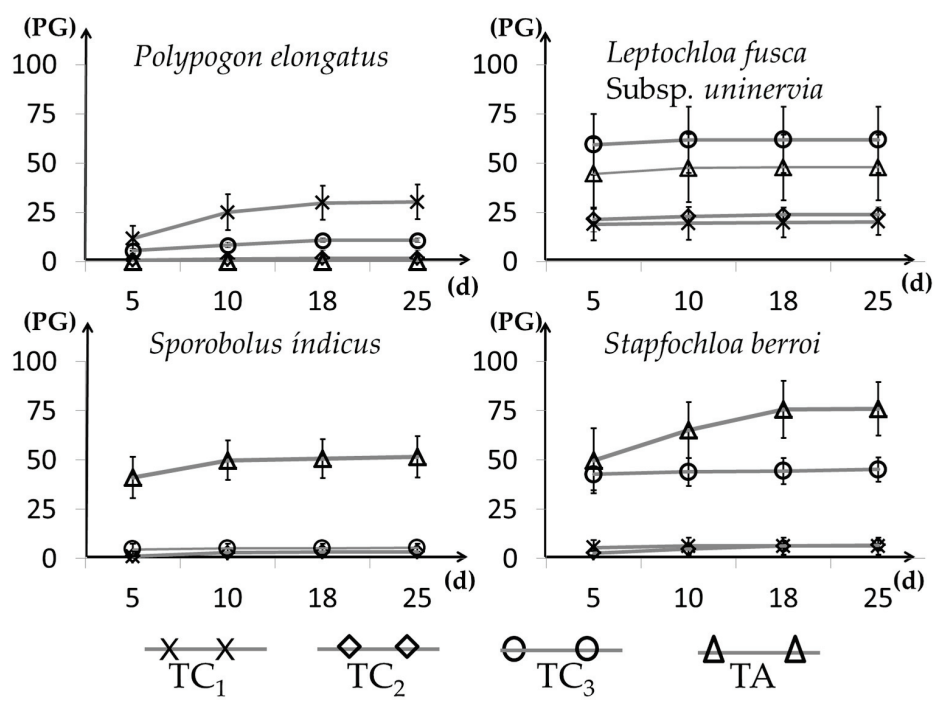

Figura 3. Evolución del porcentaje de germinación (PG en \%) registrado al día 5, 10, 18 y 25 postsiembra (d en días), bajo tres condiciones de temperaturas constantes $\left(\mathrm{TC}_{1}: 25^{\circ} \mathrm{C}\right.$, $\mathrm{TC}_{2}: 28^{\circ} \mathrm{C}, \mathrm{TC}_{3}: 37^{\circ} \mathrm{C}$ ) y un régimen de temperatura alterna (TA $20 / 35^{\circ} \mathrm{C}$ 12 horas/12 horas), en semillas de cuatro gramíneas forrajeras nativas del pastizal halofítico de la Pampa Deprimida, Argentina.

Figure 3. Evolution of germinating percentage (PG in \%) registered at 5, 10, 18 and 25 days post-sown ( $\mathrm{d}$ in days), under three constant temperature conditions $\left(\mathrm{TC}_{1}: 25^{\circ} \mathrm{C}, \mathrm{TC}_{2}: 28^{\circ} \mathrm{C}\right.$, $\mathrm{TC}_{3}: 37^{\circ} \mathrm{C}$ ) and under a alternant temperature regimen (TA $20 / 35^{\circ} \mathrm{C}$ in periods of 12 hours / 12 hours), in seeds of four native forage grasses of halophytic grassland of the Flooding Pampas, Argentina. especies estivales. En cambio, la especie invernal mantenía más de $65 \%$ de las simientes viables sin germinar al finalizar el ensayo, lo que sugiere la presencia de dormición. Es común que las simientes de las especies invernales requieran una cantidad de horas de frío (i.e., vernalización), por lo cual, la estratificación en frío suele ser una práctica frecuente para promover su germinación (Hartmann and Kester 1977). Se reportaron requerimientos de vernalización - conjuntamente con el mismo óptimo de germinación a $25^{\circ} \mathrm{C}$ - tanto para Polypogon imberbis (Phil.) Johow (Ferrari et al. 1997), como para Chascolytrum subaristatum (Lam.) Desv. (ex Briza subaristata Lam.) (Ferrari et al. 2002). Ambas son especies nativas perennes de ciclo invernal que pueden hallarse junto con $P$. elongatus tanto en la estepa de halófitas como en la pradera húmeda de mesófitas del pastizal de la Pampa Deprimida (Perelman et al. 2001). En suma, P. imberbis es una especie emparentada con P. elongatus. En la estepa de halófitas del pastizal de la Pampa Deprimida, Stapfochloa berroi, Sporobolus indi- cus y Leptochloa fusca ssp. uninervia dispersan sus semillas principalmente durante febrero, marzo y abril (Vecchio et al. en prensa a) (Figura 4). Si se tiene en cuenta el tiempo que requieren para disminuir su dormición, recién a partir de la primavera del siguiente año (i.e., octubre) podrían germinar ante condiciones externas favorables. Naturalmente, P. elongatus dispersa sus semillas durante enero y febrero, por lo que las semillas dispersadas pasan el otoño e invierno en el suelo, donde reciben la vernalización que necesitan para luego germinar en condiciones externas favorables (Figura 4).

Las tres especies estivales tuvieron mayor PG y energía germinativa (IVG) bajo el régimen de temperatura con alta amplitud térmica (TA), pero, a diferencia de las perennes, la estival anual (L. fusca ssp. uninervia) también registró alto PG e IVG incubada a una temperatura constante elevada $\left(\mathrm{TC}_{3}\right)$. Contrariamente a las estivales, la especie invernal no registró germinación con TA y alcanzó su máximo PG con

Tabla 1. Índice de velocidad de germinación (IVG), expresado como número de semillas germinadas por día \pm desvío estándar, en cuatro gramíneas forrajeras nativas del pastizal de la Pampa Deprimida, germinadas bajo tres condiciones de temperatura constantes $\left(\mathrm{TC}_{1}: 25^{\circ} \mathrm{C}, \mathrm{TC}_{2}: 28^{\circ} \mathrm{C}, \mathrm{TC}_{3}: 37^{\circ} \mathrm{C}\right.$ ) y un régimen de temperatura alterna $\left(\mathrm{TA} 20 / 35^{\circ} \mathrm{C}\right.$ en períodos de 12 horas/12 horas). Letras distintas en una misma columna indican diferencias significativas entre tratamientos de temperaturas (Tukey, $\mathrm{\square}=0.05$ ).

Table 1. Germination speed index (IVG), expressed in number of seeds germinated by day \pm standard deviation, of four native forage grasses of the grassland of the Flooding Pampa, germinated under three constant temperature conditions $\left(\mathrm{TC}_{1}: 25^{\circ} \mathrm{C}, \mathrm{TC}_{2}: 28^{\circ} \mathrm{C}, \mathrm{TC}_{3}: 37^{\circ} \mathrm{C}\right.$ ) and under an alternant temperature regimen $\left(\mathrm{TA} 20-35^{\circ} \mathrm{C}\right.$ in periods of 12 hours / 12 hours). Different letters in a same column indicate significant differences between temperature treatments (Tukey, $\mathrm{D}=0.05)$.

\begin{tabular}{lcccc}
\hline & Polypogon elongatus & Leptochloa fusca ssp uninervia & Sporobolus indicus & Stapfochloa berroi \\
\hline $\mathrm{TC}_{1}$ & $1.68 \pm 0.60 \mathrm{C}$ & $1.96 \pm 0.80 \mathrm{~A}$ & $0.34 \pm 0.16 \mathrm{~A}$ & $0.60 \pm 0.24 \mathrm{~A}$ \\
$\mathrm{TC}_{2}$ & $0.11 \pm 0.09 \mathrm{~A}$ & $2.26 \pm 0.53 \mathrm{~A}$ & $0.21 \pm 0.15 \mathrm{~A}$ & $0.44 \pm 0.24 \mathrm{~A}$ \\
$\mathrm{TC}_{3}$ & $0.66 \pm 0.33 \mathrm{~B}$ & $6.08 \pm 1.61 \mathrm{~B}$ & $0.47 \pm 0.23 \mathrm{~A}$ & $4.37 \pm 0.17 \mathrm{~B}$ \\
$\mathrm{TA}$ & $0.00 \pm 0.00 \mathrm{~A}$ & $4.65 \pm 1.73 \mathrm{~B}$ & $4.34 \pm 0.97 \mathrm{~B}$ & $6.04 \pm 0.32 \mathrm{~B}$ \\
\hline
\end{tabular}




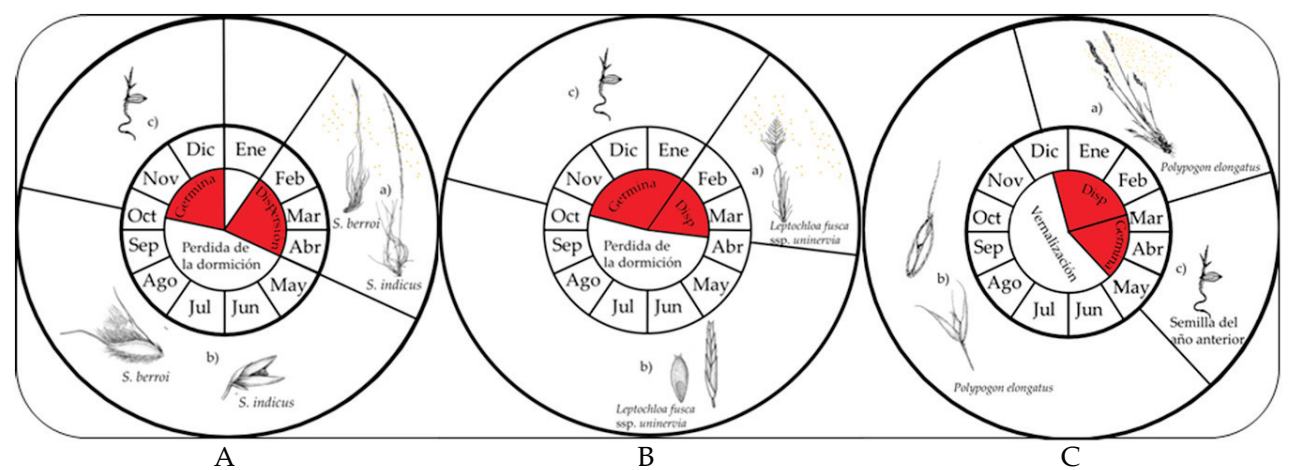

Figura 4. Ciclos de las semillas de Stapfochloa berroi y Sporobolus indicus (A), Leptochloa fusca ssp. uninervia (B) y Polypogon elongatus (C). Se indican los meses en los cuales mayormente ocurre la dispersión (a), durante los cuales la semilla pierde la dormición permaneciendo en el banco de semillas del suelo (b) y en los que se darían las mejores condiciones externas para que ocurra su germinación en la estepa de halófitas del pastizal de la Pampa Deprimida. La parte central del círculo, sombreada con rojo, indica los meses durante los cuales se recomienda orientar pruebas experimentales o decisiones de manejo del pastoreo de ganado doméstico que favorezcan los procesos de dispersión y germinación de cada especie.

Figure 4. Cycles of the seeds of Stapfochloa berroi and Sporobolus indicus (A), Leptochloa fusca ssp. uninervia (B) and Polypogon elongatus (C), indicating the months in which the dispersion mostly occurs (a), during which the seed loses its dormant stay remaining in the seed bank of the soil (b) and in which occur the best external conditions for its germination in the steppe of halophytes of the pasture of the Pampa Deprimida. The central part of the circle, shaded in red, indicates the months during which it is recommended to orient experimental tests or management decisions of domestic livestock grazing that favor the processes of dispersion and germination of each species.

la menor de las temperaturas constante $\left(\mathrm{TC}_{1}\right)$. Las semillas que requieren alta alternancia para germinar poseen mecanismos enzimáticos que funcionan a diferentes temperaturas (Vázquez-Yanes and Orozco-Segovia 1987) o bien presentan algún tipo de cubierta seminal dura e impermeable (Borges and Renas 1993). En condiciones naturales, las fluctuaciones térmicas diarias debilitan progresivamente las cubiertas, con lo que se forman microfracturas que permiten el ingreso del agua y la germinación (Taylor 1981; Bewley and Black 1982). En el caso de $S$. berroi se demostró que las glumelas que recubren el cariópside son las responsables de la dormición, ya que al quitarlas manualmente se elimina gran parte del fenómeno (Bolaños et al. 2015). En el caso de $S$. indicus, dado que en el presente estudio se utilizaron los cariópsides sin su cubierta (como naturalmente se dispersan), su respuesta a la alternancia es explicada, principalmente, por el mecanismo enzimático. Respuestas similares a la alternancia de temperatura se encontraron en otras especies perennes estivales del género Chloris (emparentado con el género Stapfochloa), tales como Chloris cuculiata Bisch. y C. subdolichostachya Muell., ambas nativas de México y propias de ambientes salinos (Herrera et al. 2006). Asimismo, nuestros resultados coinciden con lo reportado por Ferrari y Rossi (1997) y Ferrari (2008) para $S$. indicus, y con los de Altop et al. (2015) para Leptochloa fusca ssp. fascicularis [Lam.] N. Snow, especie cercanamente emparentada con L. fusca ssp. uninervia. Otras especies estivales perennes nativas del pastizal de la Pampa Deprimida, tales como Paspalum notatum Flüggé. (Marousky and West 1988), Paspalum dilatatum Poir. (Schrauf et al. 1995) y Bothriochloa laguroides (DC.) Herter (Ferrari and Rossi 1997), también presentaron requerimientos de amplitud térmica para germinar. La sensibilidad de las semillas a la amplitud térmica tiene gran importancia ecológica para la supervivencia de la especie, ya sea para adecuar la germinación a una determinada estación del año o para censar la presencia de potenciales competidores y evitar que la semilla germine en condiciones competitivamente desventajosas. Dicha sensibilidad permite a la semilla registrar la estación del año en la cual tenga lugar una determinada amplitud térmica entre el día y la noche, coincidiendo con la estación del año donde las condiciones ambientales serían las más propicias para germinar y perdurar como plántula. Además, registrar esa señal ambiental permite censar la ocupación del suelo (Thompson et al. 1977; Thompson and Grime 1983). El suelo también presenta variación en la amplitud térmica entre la fase diurna y la nocturna según la altura y la densidad del canopeo; cuanto más alto y denso sea el canopeo, menor será la temperatura máxima diurna y, en consecuencia, la amplitud térmica (Thompson et al. 1977; Benech et al. 1988).

Entre las especies estivales evaluadas, la anual L. fusca ssp. uninervia registró, además, altos valores de PG e IVG bajo la temperatura 
constante de $37^{\circ} \mathrm{C}$, y no sólo con TA como las perennes. Asimismo, la invernal ( $P$. elongatus) también reportó mayor PG y energía germinativa con una de las temperaturas constante, pero en este caso fue $25^{\circ} \mathrm{C}$. Este tipo de respuesta está asociado a especies que poseen mecanismos enzimáticos que se activan por encima de un determinado umbral térmico, lo que desata un pulso de germinación (VázquezYanes and Orozco-Segovia 1987). Dicho umbral térmico fue estudiado para Diplachne fusca (L.) Beauv (especie emparentada con L. fusca ssp. uninervia) y se reportó una ausencia de germinación con temperaturas inferiores a los $18.5^{\circ} \mathrm{C}$ y máxima germinación con $31^{\circ} \mathrm{C}$ (Morgan and Mayers 1989). En el presente estudio, L. fusca ssp. uninervia fue la única especie que tuvo un solo pulso germinativo, registrado al día 5 postsiembra, independientemente de la temperatura usada (Figura 3). No obstante, es probable que las semillas de L. fusca ssp. uninervia sean sensibles a cambios en la luz, ya que se informó fotoblastimo para L. fusca ssp. fascicularis (Altop et al. 2015); ello sería otro regulador ambiental clave de su germinación. Este tipo de requerimiento es frecuente en especies anuales cuya estrategia de vida, generalmente, es más oportunista que el de las perennes, apostando a una alta producción de semillas dispuestas a colonizar rápidamente lo claros generados en la vegetación percibidos por los cambios de luz (Thompson et al. 1993; Pons 2000). En este sentido, L. fusca ssp. uninervia es reconocida mundialmente por su capacidad para colonizar suelos sódicos o salino-sódicos (Malik et al. 1986); se la suele encontrar como especie pionera colonizando en etapas tempranas de la sucesión secundaria postdisturbio (Qadir et al. 1996) o como maleza en arroceras bajo inundación (Osca and Lidón 2011). En el caso de la invernal P. elongatus, la concentración de nitratos en solución podría estar regulando su germinación, al igual que lo reseñado para $P$. imberbis (Ferrari et al. 1997). Esto tendría una gran importancia ecológica ya que define el momento del año en el que se maximiza su germinación a campo, esto es, cuando haya cumplido sus requerimientos de vernalización, encuentre temperaturas templadas de $\sim 25{ }^{\circ} \mathrm{C}$ y una concentración elevada de nitratos en la solución del suelo. En la Pampa Deprimida, estas condiciones ocurren con mayor frecuencia durante el otoño (i.e., marzo, abril), puesto que si bien a la salida del invierno (i.e., septiembre) se cumplen las dos primeras condiciones, la concentración de nitratos en la solución del suelo es mínima (Echeverría et al. 1994). Dichos factores ambi- entales, luz en el caso L. fusca ssp. uninervia y nitratos en solución del suelo en P. elongatus, son, junto con la temperatura, reguladores externos fundamentales para la germinación de la semilla. En suma, se reportó cómo la germinación de la especie halófita Polypogon monspeliensis es sensible al estrés hídrico independientemente de la concentración de sal en el suelo (Atia et al. 2011).

Mediante los requerimientos internos y externos de la semilla, cada especie define el momento del año en el que maximiza su germinación a campo. Tanto el requerimiento de tiempo postdispersión junto con el de TA en S. berroi y S. indicus, como el relativo menor requerimiento térmico y probable fotoblastismo de L. fusca ssp. uninervia y de vernalización junto con la sensibilidad a la concentración de nitratos en $P$. elongatus, resultan de una adaptación al ambiente en el que evolucionaron. Dichos conocimientos representan un aporte valioso a la comprensión de la ecología de las especies nativas con valor forrajero que se encuentran adaptadas a ambientes con altas restricciones edáficas $\mathrm{y}$, además, constituye una oportunidad notable para orientar experimentos o decisiones de manejo del pastoreo de ganado doméstico. En la Pampa Deprimida, recién a fines de octubre se registran temperaturas mayores a $35{ }^{\circ} \mathrm{C}$, y durante parte de octubre, noviembre y diciembre suelen ocurrir amplitudes térmicas elevadas (i.e., 20 y $35^{\circ} \mathrm{C}$ ), ya que luego la temperatura mínima tiende a aumentar. Esto evidencia que, por un lado, existe una sincronía en el momento del año a partir del cual las simientes de las especies estivales dispersadas el año anterior están en condiciones de germinar; por el otro, que aumenta la ocurrencia de días con TA favorables para su germinación (Figura 4). En consecuencia, durante fines de octubre, noviembre y diciembre resultaría estratégico ensayar un descanso a fin de favorecer la germinación de $S$. berroi y $S$. indicus, siempre y cuando se haya tomado el recaudo de que el dosel del canopeo sea lo suficientemente bajo como para que favorezca el régimen de TA en la superficie del suelo. Esto se podría lograr al implementar un pastoreo intenso previo. Asimismo, en dicho manejo se debe considerar que durante febrero, marzo y abril es clave que las plantas adultas de $S$. berroi y $S$. indicus se vigoricen para poder transcurrir el invierno; en este período, además, es necesario promover la dispersión de sus semillas junto con las de L. fusca ssp. uninervia (Figura 4). Adicionalmente, dicho descanso otoñal sería 
también recomendable puesto que coincide con el que, de manera estratégica, sería recomendable para la especie invernal P. elongatus. Dicha especie invernal dispersa sus semillas durante el otoño y, al mismo tiempo, las semillas dispersadas el año anterior encuentran durante esa estación las mejores condiciones para germinar (al menos en la Pampa Deprimida, febrero, marzo y abril) (Figura 4). No obstante, en lo que respecta a experimentar manejos del pastoreo en la estepa de halófitas, las consecuencias de este estudio deberán ser corroboradas con ensayos a campo, con una adecuada escala espacial y temporal y, además, se deberá considerar la influencia de ciertas variables que regulan las condiciones microambientales del suelo, tales como el porcentaje de cobertura vegetal y broza.

Agradecimientos. Este trabajo fue realizado con el apoyo de la Universidad Nacional de la Plata, Facultad de Ciencias Agrarias y Forestales y de CONICET La Plata. Agradecemos además a la administración de los campos de la FCAyF (UNLP) y muy especialmente a los estudiantes de Agronomía C. Colillan, J. Lanz y F. Municoy por su colaboración en las mediciones. Queremos agradecer o los dos correctores, quienes desde el anonimato dieron un valioso y generoso aporte para mejorar la calidad de este manuscrito.

\section{REFERENCIAS}

Altop, E. K., H. Mennan, C. J. Phillippo, and B. H. Zandstra. 2015. Effect of the burial depth and environmental factors on the seasonal germination of bearded sprangletop (Leptochloa fusca [L.] Kunth ssp. fascicularis [Lam.] N. Snow. Weed Biology and Management 15:147-158.

Atia, A., A. Smaoui, Z. Barhoumi, C. Abdelly, and A. Debez. 2011. Differential response to salinity and water deficit stress in Polypogon monspeliensis (L.) Desf. provenances during germination. Plant Biology 13(3):541-545.

Baskin, C. C., and J. M. Baskin. 1998. Biogeography and Evolution of Dormancy and Germination. Seed Ecology. San Diego, Academic Press. Pp. 666.

Batista, W. B., R. J. C. León, and S. B. Perelman. 1988. Las comunidades vegetales de un pastizal natural de la Región de Laprida, Prov. de Buenos Aires, Argentina. Phytocoenologia 16:465-480.

Bewley, J. D., and M. Black. 1982. Physiology and biochemistry of seeds in relation to germination. Vol. II. Viability, dormancy and environmental control. Berlin: Springer Verlag. Pp. 375.

Bierhuizen, J. F., and W. A. Wagenvoort. 1974. Some aspects of seed germination in vegetables. The determination and application of heat sums and minimum temperature for germination. Sciencia Horticulturae 2:213-219.

Bolaños, V. R. A., M. C. Vecchio, and R. A. Golluscio. 2015. Dormición y tipo de suelo como determinantes en la germinación y establecimiento de Stapfochloa berroi en la Pampa Deprimida. Ecología Austral 25(1):75-80.

Borges, E. E. L., and A. B. Renas. 1993. Germinação da sementes. Pp. 83-135 in I. B. Aguiar, F. C. M. Piña-Rodrigues and M. B. Filgliolia (eds.). Sementes florestais tropicais. Abrates. Brasília, Brasil.

Burkart, S. E., León, R. J. C. and C. P. Movia. 1990. Inventario fitosociológico del pastizal de la Depresión del Salado (Prov. Bs. As.) en un área representativa de sus principales ambientes. Darwiniana 30:27-69.

Burkart, S. E., R. J. C. León, S. B. Perelman, and M. Agnusdei. 1998. The grasslands of the Flooding Pampa (Argentina): floristic heterogeneity of natural communities of the southern Salado Basin. Coenoses 13:17-27.

Burkart, S. E., M. F. Garbulsky, C. M. Ghersa, J. P. Guerchman, J. C. R. León, et al. 2005. Las comunidades potenciales del pastizal pampeano bonaerense. Pp. 379-387 in M. Oesterheld, M. Aguiar, C. Ghersa and J. Paruelo (eds.). La Heterogeneidad de la Vegetación de los Agroecosistemas. Un Homenaje a Rolando León. Facultad de Agronomía, UBA. Buenos Aires, Argentina.

Cabrera, A. L. 1994. Enciclopedia Argentina de Agricultura y Jardinería. Ed. ACME. Sta. Magdalena 635, Buenos Aires, Argentina.

Cauhépé, M., L. Hidalgo, and A. Galatoire. 1985. Aplicación de un índice de valoración zootécnica en pastizales de la Depresión del Salado. Rev Arg Prod Animal 5:681-690.

Cauhépé, M., and L. Hidalgo. 2005. La Pampa inundable: el uso ganadero como base de la sustentabilidad social, económica y ambiental. Pp. 401-411 in M. Oesterheld, M. Aguiar, C. Ghersa and J. Paruelo (eds.). La Heterogeneidad de la Vegetación de los Agroecosistemas. Un Homenaje a Rolando León. Facultad de Agronomía, UBA. Buenos Aires, Argentina.

Chaneton, E. J., S. B. Perelman, M. Omacini, and R. J. C. Léon. 2002. Grazing, environmental heterogeneity, and alien plant invasions in temperate Pampa grasslands. Biological Invasions 4:7-24.

Cole, I., I. D. Lunt, and T. Koen. 2005. Effects of sowing treatment and landscape position on establishment of the perennial tussock grass Themeda triandra (Poaceae) in degraded eucalyptus woodlands in southeastern Australia. Restoration Ecology 13:552-561.

Di Rienzo, J. A., F. Casanoves, M. G. Balzarini, L. González, M. Tablada, and C. W. Robledo. 2016. InfoStat versión 2016. Grupo InfoStat, FCA, Universidad Nacional de Córdoba, Argentina. URL: www.infostat.com.ar.

Echeverría, H., R. Bergonzi, and J. Ferrari. 1994. Estimación de la mineralización de nitrógeno en suelos del sudeste bonaerense. Boletín técnico $\mathrm{N}^{\circ} 135$. Pp. 16. Instituto Nacional de Tecnología Agropecuaria, Centro Regional Buenos 
Aires Sur, Estación Experimental Agropecuaria, Balcarce, Argentina.

Ferrari, L. 2008. Bases fisiológicas de la germinación y dormición en Sporobolus indicus (L.) R. Br. Tesis Doctoral. Facultad de Ciencias Agrícolas (UNC). Córdoba, Argentina.

Ferrari, L., E. B. Postulka, and C. López. 2002. Condiciones de germinación de semillas de Briza subaristata Lam. recolectadas en diferentes sitios de la Pampa Deprimida (Argentina). Invest Agr Prod Prot Veg 17(1):157-163.

Ferrari, L. 1999. Efecto de la temperatura y de pretratamiento en la germinación de Bothriochloa laguroides (DC.) Herter y Chaetotropis elongata (Kunth) Björkman. Rev Bras de Sem 21:84-87.

Ferrari, L., and C. A. Rossi. 1997. Seed quality of five native grasses of the humid Argentine Pampa Praire: germination condition. Pp. 19-20 in Proc. of the XVIII International Grassland Congress. University of Saskatchewan, Canada.

Hartmann, H., and D. Kester. 1977. Propagación de plantas. Principios y Prácticas. Continental México. Pp. 810.

Herrera, F., W. R. Ocumpaugh, J. A. Ortega-S, J. Lloyd-Reilley, G. A. Rasmussen, and S. Maher. 2006. Improving Germination in Windmillgrass Ecotypes. Rangeland Ecology and Management 59(6):660- 663.

International Seed Testing Association (ISTA). 1996. International Rules for Seed Testing, Zurich. Pp. 335.

Lavado, R. S., and M. A. Taboada. 1987. Soil salinization fluxes as an effect of grazing in a native grassland soil in the Flooding Pampa in Argentina. Soil, Use and Management 4:143-148.

León, R. J. C., S. Burkart, and C. Movia. 1979. Relevamiento fitosociológico del pastizal del Norte de la Depresión del Salado. Serie Fitogeográfica. INTA Buenos Aires. Pp. 17-90.

León, R. J. C., G. M. Rusch, and M. Oesterheld. 1984. Pastizales pampeanos-impacto agropecuario. Phytocoenología 12(2/3):201-218.

Maguire, J. D. 1962. Speeds of germination-aid selection and evaluation for seedling emergence and vigor. Crop Sci 2:176-183.

Malik, K. A., Z. Aslam, and M. Naqvi. 1986. Kallar grass: a plant for saline land. Nuclear Inst. Agric. Biol. (NIAB), Faisalabad, Pakistán. Pp. 56.

Marousky, F. J., and S. H. West. 1988. Germination of bahiagrass in response to temperature and scarification. Journal of the American Society for Horticultural Science 113(6):845-849.

Mayer, A. M., and A. Poljakoff-Mayber. 1989. The Germination of Seeds. Pergamon Press Ltd.

Mollard, F. P. O. 2007. Regeneración de pastizales naturales por germinación de semillas: control del estado de dormición de las semillas por el contenido hídrico del suelo. Tesis doctoral. Escuela para Graduados Alberto Soriano, FAUBA. Buenos Aires, Argentina. Pp. 108.

Morgan, W. C., and B. A. Myers. 1989. Germination of the salt-tolerant grass Diplachne fusca L. 1. Dormancy and temperature responses. Austr J Bot 37:225-237.

Osca, J. M., and A. Lidón. 2011. Estudio del banco de semillas de Leptochloa fusca ssp. Uninervia y L. fusca ssp. Facicularis en arrozales de Valencia. XII Congreso de la Sociedad Española de Malherbología. La Laguna. España. Pp. 83-86.

Otondo, J. 2011. Efectos de la introducción de especies megatérmicas sobre características agronómicas y edáficas de un ambiente halomórfico de la Pampa Inundable. Tesis Magister en Recursos Naturales. Escuela para Graduados Alberto Soriano. FAUBA, Buenos Aires, Argentina. Pp. 100-136.

Perelman, S., R. J. C. León, and V. A. Deregibus. 1982. Aplicación de un método objetivo al estudio de las comunidades de pastizal de la Depresión del Salado (Provincia de Buenos Aires). Revista Facultad de Agronomía de Buenos Aires 3:27-40.

Perelman S. B., R. J. C. León, and M. Oesterheld. 2001. Cross-Scale vegetation patterns of Flooding Pampa grasslands. J Ecology 89:562-577.

Phillips, R. E. 1984. Soil moisture. Pp. 66-86 in R. E. Phillips and S. H. Phillips (eds.). No-tillage agriculture. Principles and practices. Van Nostrand Reinhold Co., New York, USA.

Pons, T. 2000. Seed responses to light. Pp. 237-260 in M. Fenner (ed.) Seeds: The ecology of Regeneration in Plants Communities. CAB International, Wallingford, UK.

Probert, R. 2000. The role of temperature in germination ecophysiology. Pp. 261-292 in M. Fenner (ed.). Seeds: The Ecology of Regeneration in Plant Communities. CAB International, Wallingford, UK.

Puhl, L. E., S. B. Perelman, W. B. Batista, S. E. Burkart, and R. J. C. Léon. 2014. Local and regional long-term diversity changes and biotic homogenization in two temperate grasslands. Journal of Vegetation Science 25:1278-1288.

Qadir, M., R. H. Qureshi, and N. Ahmad. 1996.Reclamation of a saline-sodic soil by gypsum and Leptochloa fusca L. Geoderma 74:207-217.

Quiroga, E., L. Blanco, and E. Orionte. 2009. Evaluación de estrategias de rehabilitación de pastizales áridos. Ecología Austral 19(2):107-117.

Rearte, D. H. 1997. La integración de la ganadería argentina. SAGPyA-INTA. Buenos Aires, Argentina. Pp. 48.

Rees, M. 1997. Seed dormancy. Pp. 214-238 in M. Crawley (ed.). Plant Ecology. Blackwell Science. London, England.

Ruiz, M. de los A., M. A. Pérez, J. A. Argüello, and F. J. Babinec. 2003. Madurez fisiológica de la semilla de Bromus auleticus Trin. (cebadilla chaqueña). RIA 32(2):3-19.

Schrauf, G. E., P. S. Cornaglia, V. A. Deregibus, and M. G. Rissola. 1995. Improvement germination behavior of Paspalum dilatatum Poir. seeds under different pre-conditioning treatments. New Zealand Journal of Agricultural Research 38(3):501-509.

Shaidaee, G., B. E. Dahl, and R. M. Hansen. 1969. Germination and emergence of different age seed of six grasses. J of 
Ran Manage 22:240-245.

Snyman, H. A. 2003. Revegetation of bare patches in a semi-arid rangeland of South Africa: an evaluation of various techniques. Journal of Arid Environments 55:417-432.

Soriano, A. 1991. Río de la Plata Grasslands. Ecosystems of the World a Natural Grasslands. Introduction and Western Hemisphere. Cap. 19. Pp. 367-407 in R. T. Coupland (ed.). Elsevier. New York. EE.UU.

Taylor, G. B. 1981. Effect of constant temperature treatments followed by fluctuating temperatures on the softening of hard seeds of Trifolium subterraneum L. Australian Journal of Plant Physiology 8(2):547-558.

Thompson, K., J. P. Grime, and G. Mason. 1977. Seed germination in response to diurnal fluctuations of temperature. Nature 267:147-149.

Thompson, K., and J. P. Grime. 1983. A comparative study of germination response to diurnally fluctuating temperatures. Journal of Applied Ecology 20:141-156.

Thompson, K., S. R. Band, and J. Hodgson. 1993. Seed size and shape predict persistence in soil. Funct Ecol 7:236241.

Vázquez-Yanes, C., and A. Orozco-Segovia. 1987. Fisiología ecológica de semillas en la Estación de Biología Tropical "Los Tuxtlas", Veracruz, México. Revista de Biología Tropical 35(1):85-96.

Vecchio, M. C., R. Golluscio, A. M. Rodríguez, and V. R. A. Bolaños. 2019. Rotational grazing improves grassland condition of the halophytic steppe in Flooding Pampa (Argentina). The Rangeland Journal 41(1):1-12.

Vecchio, M. C., R. Golluscio, A. M. Rodríguez, and M. A Taboada. 2018. Improvement of saline- sodic grassland soils properties by rotational grazing in Argentina. Rangeland Ecology and Management 71(6):807-814.

Vervoorst, F. B. 1967. Las comunidades vegetales de la Depresión del Salado (Provincia de Buenos Aires). In La vegetación de la República Argentina. Serie Fitogeográfica N 7. INTA. Pp. 259.

Yoon, S., S. Muruyama, and S. Kosaka. 1985. Studies on temperature responses of grasses. Comparison on germination of temperate and tropical grasses in various temperature conditions. Journal of the Yamagata Agriculture and Forestry Society 42(1):21-26.

Zuloaga, F. O., O. Morrone, and M. Belgrano. 2008. Catálogo de las Plantas Vasculares del Cono Sur. Argentina, Sur de Brasil, Chile, Paraguay y Uruguay. Monographs in Systematic Botany from the Missouri Botanical Garden 107, 3 volumes. Pp. 3486. URL: www.darwin.edu.ar. 